\title{
Epigallocatechin-3-gallate Enhances Radiation Sensitivity in Colorectal Cancer Cells Through Nrf2 Activation and Autophagy
}

\author{
TUMENJIN ENKHBAT ${ }^{1}$, MASAAKI NISHI ${ }^{1}$, KOZO YOSHIKAWA $^{1}$, HIGASHIJIMA JUN $^{1}$, \\ TAKUYA TOKUNAGA ${ }^{1}$, CHIE TAKASU $^{1}$, HIDEYA KASHIHARA ${ }^{1}$, \\ DAICHI ISHIKAWA ${ }^{1}$, MASAHIDE TOMINAGA ${ }^{2}$ and MITSUO SHIMADA ${ }^{1}$ \\ ${ }^{1}$ Department of Surgery, Institute of Biomedical Sciences, \\ Tokushima University Graduate School, Tokushima, Japan; \\ ${ }^{2}$ Department of Therapeutic Radiology Institute of Biomedical Sciences, \\ The University of Tokushima Graduate School, Tokushima, Japan
}

\begin{abstract}
Background/Aim: Epigallocatechin-3-gallate (EGCG) is a major polyphenolic component of green tea. EGCG plays a potential role in radio-sensitizing cancer cells. The combined effect of EGCG and radiation was investigated in a colorectal cancer cell line, focusing on nuclear factor (erythroid-derived 2)-like 2 (Nrf2) autophagy signalling. Materials and Methods: HCT-116 cells were treated with $12.5 \mu M$ EGCG for different periods of time, 2 Gy radiation, or both. Cell viability was determined with the WST-8 assay. The number of colonies was determined with the colony formation assay. mRNA expression of LC3 and caspase-9 was analyzed with quantitative real-time polymerase chain reaction. Results: Combination treatment with EGCG and radiation significantly decreased the growth of HCT-116 cells. The number of colonies was reduced to $34.2 \%$ compared to the control group. Immunofluorescence microscopy images showed that nuclear translocation of Nrf2 was significantly increased when cells were treated with the combination of EGCG and radiation compared to the control and single-treatment groups. Combined treatment with EGCG and radiation significantly induced LC3 and caspase-9 mRNA expression. Conclusion: EGCG increased the sensitivity of colorectal cancer cells to radiation by inhibiting cell proliferation and inducing Nrf2 nuclear translocation and autophagy.
\end{abstract}

Correspondence to: Mitsuo Shimada, MD, Ph.D., FACS, Department of Surgery, Institute of Health Biosciences, University of Tokushima Graduate School 3-18-15, Kuramoto-cho, Tokushima, 770-8503 Japan. Tel: +81 886337137, Fax: +81 886319698, e-mail: mitsuo.shimada@tokushima-u.ac.jp

Key Words: EGCG, radiation, colorectal cancer, Nrf2, autophagy.
Radiotherapy (RT) is commonly used for the effective treatment of solid tumours, including colorectal cancer (CRC) (1). RT induces DNA damage and cytotoxicity by generating reactive oxygen species inside the tumour cells (2). However, RT becomes ineffective due to radio-resistance of tumour cells (3), thus, improvement of the anti-tumour effect of RT on cancer cells is required in the clinical setting. Green tea, one of the most widely consumed beverages worldwide, is rich (30\% of dry weight) in non-oxidised components called catechins (4). Epigallocatechin-3-gallate (EGCG) is a major polyphenolic substance found in green tea. We have previously reported the beneficial effects of $\operatorname{EGCG}(5,6)$. This compound inhibits cancer progression in vitro and in vivo, not only during carcinogenesis, but also during the progression and metastatic stages of various types of malignancies (7). Furthermore, EGCG has a potential role as a radio-sensitizer of cancer cells and has shown some synergistic effects with other factors in some preliminary studies (8). However, little is known about the precise cancer-preventing mechanism of the combination therapy of EGCG and RT in CRC.

Nuclear factor erythroid 2-related factor 2 (Nrf2) is a key transcription factor that regulates antioxidant and detoxification enzymes (9). The Nrf2/keap1 autophagy pathway plays a pivotal role in cancer cell death (10). EGCG is a major inducer of Nrf2 (11). We hypothesised that EGCG and radiation synergistically induce Nrf2 and autophagy.

The aim of this study was to investigate the combined effect of EGCG and radiation on a CRC cell line, focusing in particular on Nrf2 activity and autophagy.

\section{Materials and Methods}

Cell culture and reagents. The colon cancer cell line HCT-116 was purchased from the American Type Culture Collection (Manassas, 
VA, USA). HCT-116 cells were maintained as a monolayer in McCoy's 5A medium (Gibco, Life Technologies, Tokyo, Japan) supplemented with $10 \%$ foetal bovine serum, penicillin $(100 \mathrm{U} / \mathrm{ml})$, and streptomycin $(100 \mu \mathrm{g} / \mathrm{ml})$ in a humidified atmosphere of $5 \%$ $\mathrm{CO}_{2}$ at $37^{\circ} \mathrm{C}$. EGCG was purchased from Bio Verde (Kyoto, Japan) and dissolved in $1 \times$ phosphate-buffered saline (PBS) (6).

Cell proliferation assays. Cell proliferation was assessed with the WST-8 assay using Cell Counting Kit-8 (CCK-8) (Dojindo Laboratories, Kumamoto, Japan). HCT-116 cells $\left(5 \times 10^{3} /\right.$ well $)$ were seeded in 96-well plates and incubated for $24 \mathrm{~h}$. Cells were incubated with or without $12.5 \mu \mathrm{M}$ EGCG for $48 \mathrm{~h}$ and simultaneously exposed to 2 Gy radiation in McCoy's 5A medium. Control cells were handled similarly to the cells treated with EGCG and/or radiation. Each treatment was carried out in triplicate. After incubation, $10 \mu \mathrm{lCCK}-8$ reagent was added to each well, and the plates were incubated at $37^{\circ} \mathrm{C}$ in an atmosphere of $5 \% \mathrm{CO}_{2}$ for $4 \mathrm{~h}$. The optical density was then measured with a microplate reader (Multiskan JX; MTX Lab Systems, Vienna, VA, USA) at a wavelength of $450 \mathrm{~nm}(6)$.

$X$-ray irradiation. X-ray irradiation was carried out with a HITACHI MBR-1520R-3 (Hitachi Power Solutions Co., Ltd., Shizuoka, Japan), which was operated at $150 \mathrm{kVp}$ and $20 \mathrm{~mA}$ at room temperature. The dose rate was $4.31 \mathrm{~Gy} / \mathrm{min}$.

Colony formation assay. HCT-116 cells were treated with or without 12.5 $\mu \mathrm{M}$ EGCG for $24 \mathrm{~h}$ followed by 2 -Gy irradiation. Cells were then trypsinised and counted. 50 cells were seeded in 30-mm diameter Petri dishes. Cells were treated with or without $12.5 \mu \mathrm{M}$ EGCG for $1 \mathrm{~h}$ later, and then cultured for 9-14 days. Cells were fixed with glutaraldehyde $(6.0 \% \mathrm{v} / \mathrm{v})$ and stained with crystal violet. Colonies containing more than 50 cells were counted under a stereomicroscope. Each experiment was performed in triplicate. Error bars represent the mean $\pm \mathrm{SD}$ of at least three indepedent experiments.

Quantitative real-time polymerase chain reaction ( $q R T-P C R$ ). After treatment with EGCG for $48 \mathrm{~h}$ and/or radiation, total RNA was extracted from cells using the RNeasy Mini Kit (Qiagen, Hilden, Germany). cDNA was synthesised from $2.5 \mu \mathrm{g}$ total RNA by reverse transcription using the Super Script RT kit (Promega, Madison, WI, USA) according to the manufacturer's instructions. qRT-PCR was performed using the Applied Biosystems 7500 realtime PCR system, TaqMan Gene Expression Assays on demand, and TaqMan Universal Master Mix (gene specific TaqMan probes on a StepOne Plus; Applied Biosystems, Foster City, CA, USA). Human CASPASE-9 (Hs00154261_m1) and LC3 (Hs01076567_g1) TaqMan primers were used. GAPDH was used as an internal control for normalization. Expression levels of all genes were calculated as a ratio to $G A P D H$. Amplification data were analysed with an Applied Biosystems Prism 7500 Sequence Detection System version 1.3.1 (Applied Biosystems) (6). Error bars represent the mean \pm SD of at least three independent experiments.

Immunofluorescence. Colorectal carcinoma cells were plated on a glass base dish $(27 \mathrm{~mm} \varnothing)$ at a density of $1 \times 10^{6}$ cells per dish. Following the indicated treatments, cells were washed with PBS and fixed for $30 \mathrm{~min}$ with $4 \%$ paraformaldehyde in PBS. Next, cell membranes were permeabilised by treatment with $0.1 \%$ Triton X-100 in PBS for 2 min. After blocking with 3\% bovine

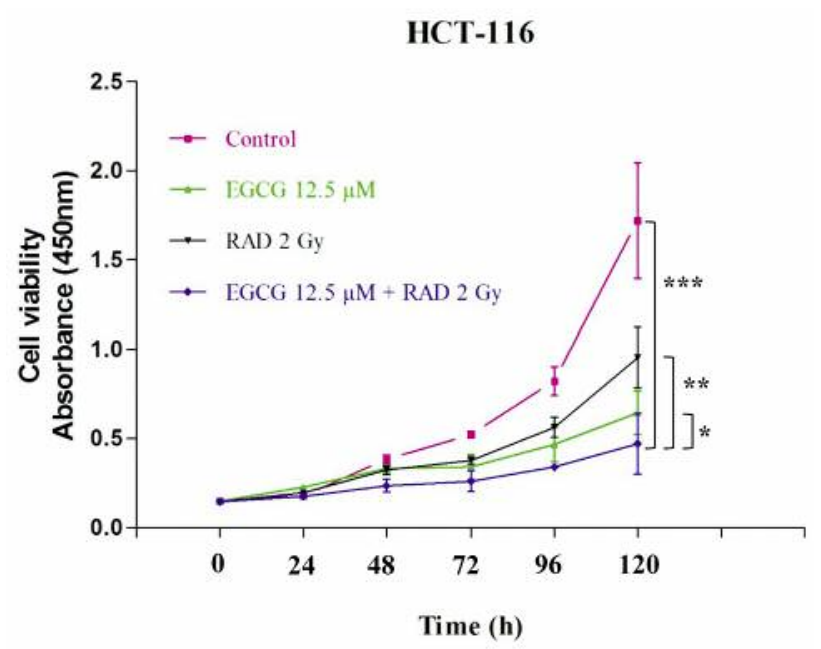

Figure 1. Growth inhibition of the human colon cancer cell line, HCT116, treated with $12.5 \mu M$ EGCG, 2 Gy radiation, or both. Combination treatment with EGCG and radiation significantly decreased the growth of HCT-116 cells compared with control, EGCG only-, or radiation only-treated cells. Data are the mean \pm standard error of the mean of three independent experiments. ${ }^{*} p<0.05 ; * * p<0.01 ; * * *<0.001$.

serum albumin in PBS for $1 \mathrm{~h}$ at room temperature, cells were treated with primary antibodies (1:200) in blocking buffer for 1 h. Goat anti-rabbit Alexa Fluor 555 was used as the secondary antibody (1:1000). Cell nuclei were stained with $5 \mu \mathrm{g} / \mathrm{ml}$ DAPI sc-3598 (Invitrogen Corp, Carlsbad, CA, USA) for $10 \mathrm{~min}$. After washing, immunofluorescence images were photographed with a BZ-x700 fluorescence microscope (Keyence, Osaka, Japan). Error bars represent the mean $\pm S D$ of at least three independent experiments.

Statistical analysis. Data are expressed as the mean \pm standard error of the mean. Statistical analyses were performed using SPSS for Windows version 17.0 (SPSS Inc., Chicago, IL, USA). Statistical significance was determined at $p<0.05$.

\section{Results}

EGCG increased sensitivity to radiation and reduced cell proliferation. The CCK-8 cell proliferation assay was used to evaluate the effects of EGCG, radiation, and their combination at different time periods on the viability of the colon cancer cell line HCT-116. Combination treatment with EGCG and radiation significantly decreased the growth of HCT-116 cells compared with the control, EGCG only, or radiation only treated cells $(p<0.05$; Figure 1$)$. In the colony formation assay, EGCG only and radiation only decreased the colony number to $77.1 \%$ and $55.2 \%$, respectively, compared to the control. Combination treatment with EGCG and radiation significantly decreased the colony number to $34.2 \%$ of the control (Figure 2). 
A

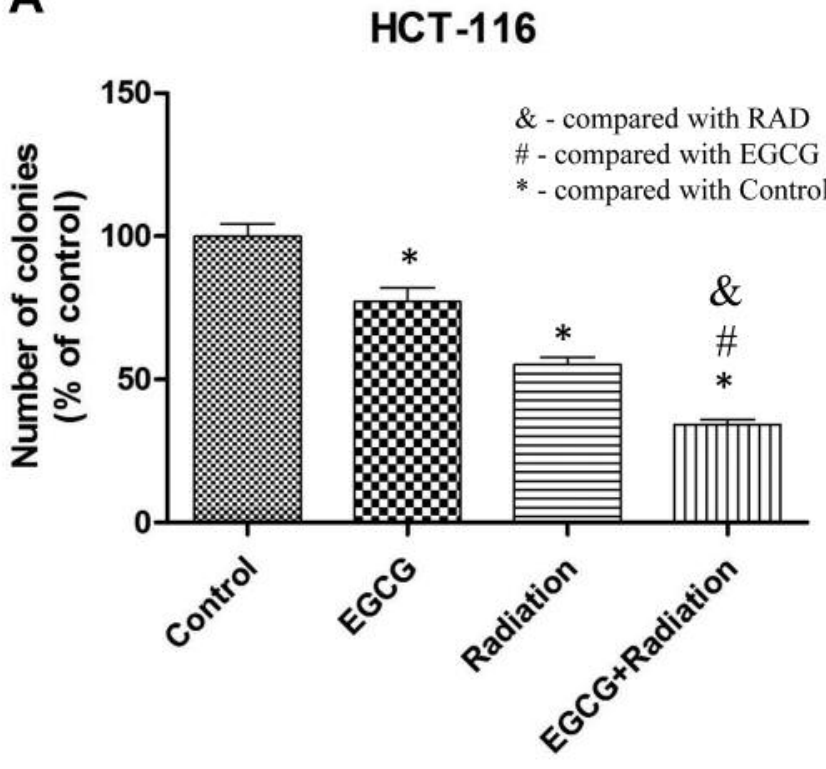

B

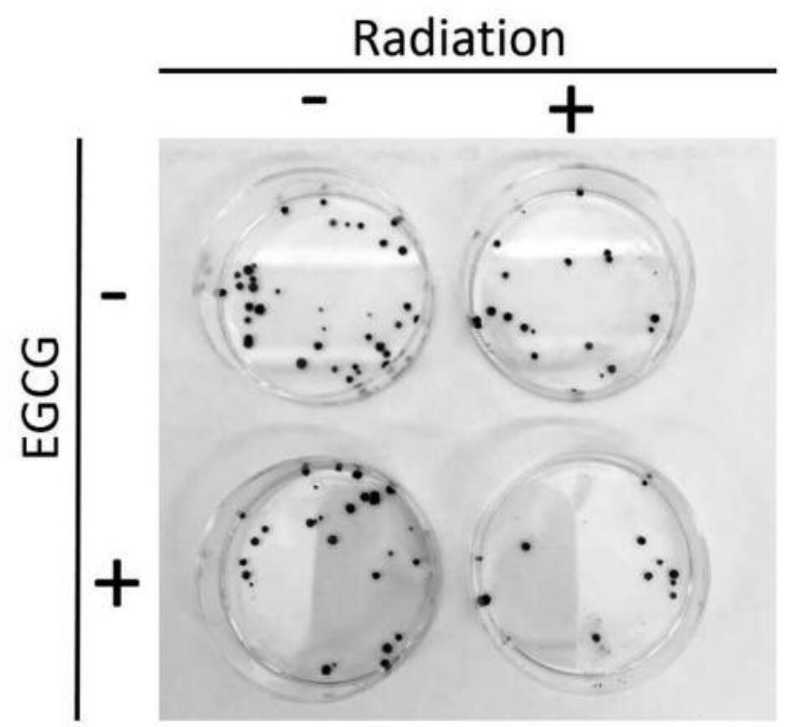

Figure 2. Combination treatment with $12.5 \mu M$ EGCG and 2-Gy radiation significantly decreased the number of colonies of HCT-116 cells. Quantified data (A) and representative images $(B)$ of the clonogenic growth experiment of HCT-116 cells. Colonies were counted after 2 weeks. *Indicates a significant difference compared to control; \#Indicates a significant difference compared to EGCG only; \&Indicates a significant difference compared to radiation only $(p<0.05)$.

Combination treatment with EGCG and radiation enhanced expression of proteins and $m R N A s$ related to autophagy and apoptosis. Immunofluorescence microscopy images showed that the nuclear translocation of Nrf2 was significantly increased by combination treatment with EGCG and radiation compared with the control and single treatment groups (Figure 3).

qRT-PCR analysis showed that combination treatment with EGCG and radiation significantly induced $L C 3$ (Figure 4A) and caspase-9 mRNA expression (Figure 4B).

\section{Discussion}

In this study, the effects of combination treatment with EGCG and radiation in CRC cell growth and underlying mechanisms were investigated. Combination treatment with EGCG and radiation inhibited CRC cell growth, and induced nuclear translocation of $\mathrm{Nrf} 2$ and the expression of autophagy-related genes.

EGCG is a well-known scavenger of reactive oxygen species and acts as an antioxidant through modulation of transcription factors and enzyme activity (5). We previously reported that EGCG stimulates liver regeneration and improves liver damage after massive hepatectomy via antioxidative and anti-inflammatory effects (5). EGCG also has an anti-tumour effect as it inhibits cancer cell proliferation, induces cell death and cell cycle arrest, inhibits invasion and metastasis, and suppresses angiogenesis and self-renewal in hepatoma and colon cancer stem cells $(6,12-14)$.

Some reports have suggested beneficial effects of EGCG and RT in cancer treatment (15-16). Combination treatment with EGCG and radiation induced apoptosis in a leukaemia cell line (15). Zhang demonstrated that EGCG administration and RT reduced serum vascular endothelial growth factor, hepatocyte growth factor, and the activation of metalloproteinase- 9 and metalloproteinase- 2 in breast cancer patients (16). Clinical trials testing the efficacy of the combination therapy are now ongoing $(17,18)$. However, to the best of our knowledge, the precise mechanism of action of EGCG is unknown, but may involve the Nrf2 autophagy pathway.

Nrf2 is a transcription factor with multiple roles, that was described as a paradoxical protein with controversial suppressing and promoting roles in cancer cells, depending on its intracellular location $(19,20)$. As a tumour suppressor, Nrf2 is important in chemoprevention by activating the main cellular defence mechanism against metabolic, xenobiotic, and oxidative stress. Nrf2 loss is related to enhanced metastasis. On the other hand, Nrf2 functions as an oncogene. Nrf2 constitutive activation is present in many tumour types and Nrf2 hyperactivation favours cancer cell survival and promotes cell growth (20).

The Nrf2-keap1-P62 pathway promotes autophagy (10), which is a fundamental cellular degradation mechanism that 

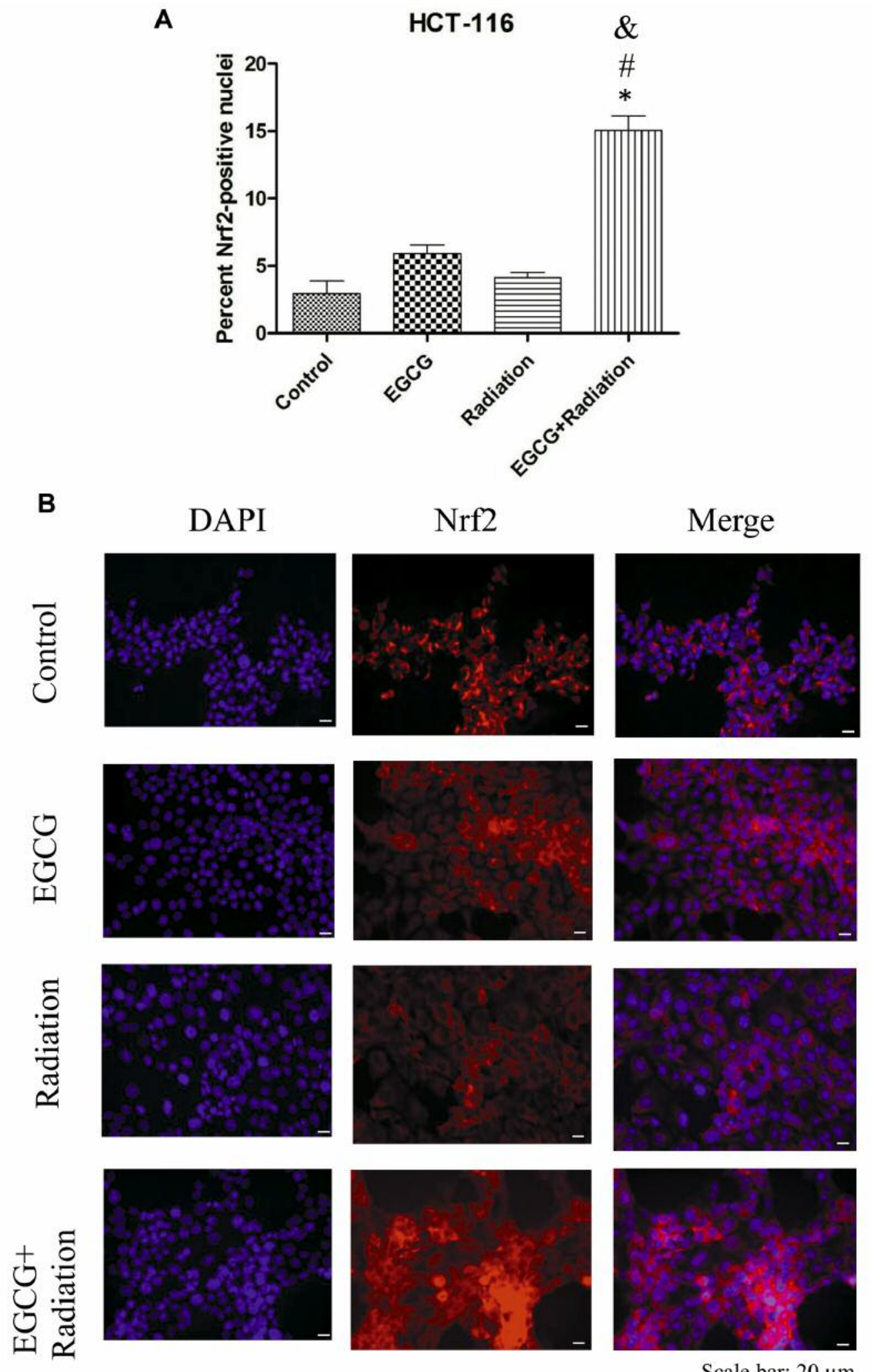

Scale bar: $20 \mu \mathrm{m}$.

Figure 3. Combination treatment with EGCG and radiation significantly increased the translocation of Nrf2 protein into the nuclei. Cells were treated with $12.5 \mu M$ EGCG and/or 2 Gy radiation for $48 \mathrm{~h}(\mathrm{~A}, \mathrm{~B})$. *Indicates a significant difference compared to control; \#Indicates a significant difference compared to EGCG only; ${ }^{\circledR}$ Indicates a significant difference compared to radiation only $(p<0.05)$. 

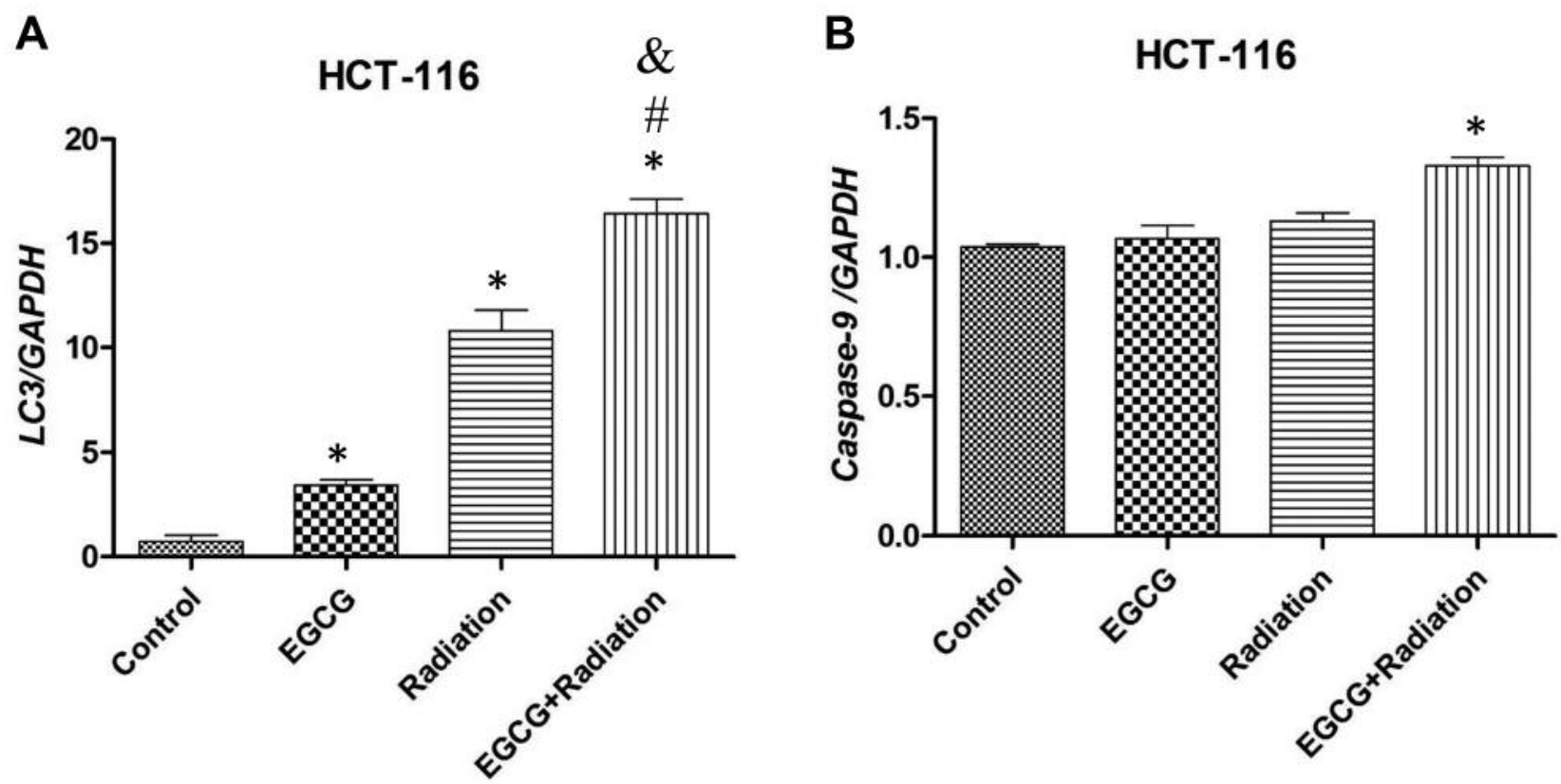

Figure 4. Effects of EGCG only, radiation only, or both on mRNA expression of LC3 and caspase-9 in HCT-116 cells. qRT-PCR analysis of mRNA expression of $L C 3(A)$ and caspase-9 (B). *Indicates a significant difference compared to control; "Indicates a significant difference compared to EGCG only; \&Indicates a significant difference compared to radiation only $(p<0.05)$.

maintains tissue homeostasis. Autophagy is classified into three types: 1. macro-autophagy, 2. micro-autophagy, and 3. mitophagy. Mitophagy is a selective type of macro-autophagy in which mitochondria are selectively targeted for degradation. Mitophagy can have the beneficial effect of eliminating old and/or damaged mitochondria. In the tumour microenvironment, induced mitophagy could exert anti-tumour effects via clearance of dysfunctional mitochondria in cancer cells $(21,22)$.

EGCG is a major Nrf2 inducer (11). Another Nrf2 inducer, sulforaphene, stimulates nuclear retention of $\mathrm{Nrf} 2$ and activates AMP kinase to induce mammalian target of rapamycin signalling, leading to mitophagic cell death in lymphoma cells (23). In our experiment, combination treatment with EGCG and radiation significantly induced nuclear translocation of $\mathrm{Nrf} 2$ and up-regulated $L C 3$ and caspase- 9 mRNA expression. These findings suggested that combination treatment with EGCG and radiation may induce mitophagic cell death.

In conclusion, EGCG increased the sensitivity of CRC cells to radiation by inhibiting cell survival, inducing Nrf2 nuclear translocation and up-regulating autophagy-related genes.

\section{Acknowledgements}

The Authors are grateful to the members of the Department of Surgery, Institute of Health Biosciences at The University of Tokushima for their important contributions to this study.

\section{References}

1 Haraldsdottir S, Einarsdottir HM, Smaradottir A, Gunnlaugsson A and Halfdanarson TR: Colorectal cancer - review. Laeknabladid 100(2): 75-82, 2014.

2 Chen HHW and Kuo MT: Improving radiotherapy in cancer treatment: Promises and challenges. Oncotarget 8(37): 6274262758, 2017.

3 Lee SL, Ryu H, Son AR, Seo B, Kim J, Jung SY, Song JY, Hwang SG and Ahn J: TGF-beta and hypoxia/reoxygenation promote radioresistance of A549 lung cancer cells through activation of Nrf2 and EGFR. Oxid Med Cell Longev 2016: 6823471, 2016.

4 Yang CS, Wang H, Li GX, Yang Z, Guan F and Jin H: Cancer prevention by tea: Evidence from laboratory studies. Pharmacol Res 64(2): 113-122, 2011.

5 Wubetu GY, Shimada M, Morine Y, Ikemoto T, Ishikawa D, Iwahashi S, Yamada S, Saito Y, Arakawa Y and Imura S: Epigallocatechin gallate hinders human hepatoma and colon cancer sphere formation. J Gastroenterol Hepatol 31(1): 256264, 2016.

6 Saito Y, Mori H, Takasu C, Komatsu M, Hanaoka J, Yamada S, Asanoma M, Ikemoto T, Imura S, Morine Y, Utsunomiya T and Shimada M: Beneficial effects of green tea catechin on massive hepatectomy model in rats. J Gastroenterol 49(4): 692-701, 2014.

7 Singh BN, Shankar S and Srivastava RK: Green tea catechin, epigallocatechin-3-gallate (EGCG): mechanisms, perspectives and clinical applications. Biochem Pharmacol 82(12): 18071821, 2011. 
8 Suganuma M, Saha A and Fujiki H: New cancer treatment strategy using combination of green tea catechins and anticancer drugs. Cancer Sci 102(2): 317-323, 2011.

9 DeNicola GM, Karreth FA, Humpton TJ, Gopinathan A, Wei C, Frese K, Mangal D, Yu KH, Yeo CJ, Calhoun ES, Scrimieri F, Winter JM, Hruban RH, Iacobuzio-Donahue C, Kern SE, Blair IA and Tuveson DA: Oncogene-induced Nrf2 transcription promotes ROS detoxification and tumorigenesis. Nature 475(7354): 106-109, 2011.

10 Ichimura Y, Waguri S, Sou YS, Kageyama S, Hasegawa J, Ishimura R, Saito T, Yang Y, Kouno T, Fukutomi T, Hoshii T, Hirao A, Takagi K, Mizushima T, Motohashi H, Lee MS, Yoshimori T, Tanaka K, Yamamoto $\mathrm{M}$ and Komatsu $\mathrm{M}$ : Phosphorylation of p62 activates the Keap1-Nrf2 pathway during selective autophagy. Mol Cell 51(5): 618-631, 2013.

11 Sun W, Liu X, Zhang H, Song Y, Li T, Liu X, Liu Y, Guo L, Wang $\mathrm{F}$, Yang T, Guo W, Wu J, Jin $\mathrm{H}$ and $\mathrm{Wu} \mathrm{H}$ : Epigallocatechin gallate upregulates NRF2 to prevent diabetic nephropathy via disabling KEAP1. Free Radic Biol Med 108: 840-857, 2017.

12 Paschka AG, Butler R and Young CY: Induction of apoptosis in prostate cancer cell lines by the green tea component, (-)epigallocatechin-3-gallate. Cancer Lett 130(1-2): 1-7, 1998.

13 Tan X, Hu D, Li S, Han Y, Zhang Y and Zhou D: Differences of four catechins in cell cycle arrest and induction of apoptosis in LoVo cells. Cancer Lett 158(1): 1-6, 2000.

14 Masuda M, Suzui $M$ and Weinstein IB: Effects of epigallocatechin-3-gallate on growth, epidermal growth factor receptor signaling pathways, gene expression, and chemosensitivity in human head and neck squamous cell carcinoma cell lines. Clin Cancer Res 7(12): 4220-4229, 2001.

15 Baatout S, Derradji H, Jacquet P and Mergeay M: Increased radiation sensitivity of an eosinophilic cell line following treatment with epigallocatechin-gallate, resveratrol and curcuma. Int J Mol Med 15(2): 337-352, 2005.
16 Zhang G, Wang Y, Zhang Y, Wan X, Li J, Liu K, Wang F, Liu K, Liu Q, Yang C, Yu P, Huang Y, Wang S, Jiang P, Qu Z, Luan J, Duan H, Zhang L, Hou A, Jin S, Hsieh TC and Wu E: Anti-cancer activities of tea epigallocatechin-3-gallate in breast cancer patients under radiotherapy. Curr Mol Med 12(2): 163-176, 2012.

17 Zhao H, Xie P, Li X, Zhu W, Sun X, Sun X, Chen X, Xing L and $\mathrm{Yu}$ J: A prospective phase II trial of EGCG in treatment of acute radiation-induced esophagitis for stage III lung cancer. Radiother Oncol 114(3): 351-356, 2015.

18 Zhao H, Zhu W, Jia L, Sun X, Chen G, Zhao X, Li X, Meng X, Kong L, Xing L and Yu J: Phase I study of topical epigallocatechin3-gallate (EGCG) in patients with breast cancer receiving adjuvant radiotherapy. Br J Radiol 89(1058): 20150665, 2016.

19 Gonzalez-Donquiles C, Alonso-Molero J, Fernandez-Villa T, Vilorio-Marqués L, Molina AJ and Martín V: The NRF2 transcription factor plays a dual role in colorectal cancer: A systematic review. PLoS One 12(5): e0177549, 2017.

20 Menegon S, Columbano A and Giordano S: The dual roles of NRF2 in cancer. Trends Mol Med 22(7): 578-593, 2016.

21 Vyas S, Zaganjor E and Haigis MC: Mitochondria and Cancer. Cell 166(3): 555-566, 2016.

22 Ding WX and Yin XM: Mitophagy: mechanisms, pathophysiological roles, and analysis. Biol Chem 393(7): 547-564, 2012.

23 Wang H, Wang F, Wu S, Liu Z, Li T, Mao L, Zhang J, Li C, Liu $\mathrm{C}$ and Yang Y: Traditional herbal medicine-derived sulforaphene promotes mitophagic cell death in lymphoma cells through CRM1-mediated p62/SQSTM1 accumulation and AMPK activation. Chem Biol Interact 281: 11-23, 2018.

Received October 4, 2018

Revised October 22, 2018

Accepted October 23, 2018 